\title{
Special issue: Precision Polymer Synthesis
}

\author{
Makoto Ouchi $^{1} \cdot$ Christine K. Luscombe $^{2} \cdot$ Junpo $\mathrm{He}^{3} \cdot$ Keiji Tanaka $^{4}$
}

Received: 24 September 2019 / Accepted: 24 September 2019 / Published online: 6 January 2020

(c) The Society of Polymer Science, Japan 2020

Since 1920, when Staudinger first reported his seminal paper describing evidence that macromolecules exist, many researchers have studied synthetic methodologies of polymers and developed their practical applications over the last 100 years. In particular, triggered by great discoveries in the 1950 s, that is stereospecific polymerization of propylene by Natta and living anionic polymerization of styrene by Szwarc, precise control of the primary structure of polymers has attracted attention because the control could lead to tough/lightweight plastics and elastomers that are now essential in our daily lives. Many people have concentrated their efforts to the breakthrough of precision polymer synthesis, leading to recent progress over the last few decades, such as chain-growth polycondensation [1] and metalcatalyzed reversible deactivation radical polymerization [2]. The precision methodology has also allowed for advanced control over polymer chain structures, such as the helical [3] and topological [4] structures.

With the development of technologies related to polymers, demands for polymer synthesis are increasingly diverse and challenging. To approach these, an emergence of new methodologies allowing advanced control for existing polymers, as well as syntheses of new type of polymers, is expected. Sequence-controlled polymerization is one such example where researchers are thriving to achieve smarter polymers similar to those seen in nature [5].

It is with great pleasure that we publish this special issue entitled "Precision Polymer Synthesis" in 2020 after
100 years of Staudinger's first claim. We invited outstanding researchers all over the world to the issue and collected 14 original articles, 3 focus reviews and 1 review. We believe that this special issue will be valuable for many researchers towards next 100 years in polymer science. Finally, we greatly appreciate all the authors and referees for their contributions to the special issue.

\section{Compliance with ethical standards}

Conflict of interest The authors declare that they have no conflict of interest.

Publisher's note Springer Nature remains neutral with regard to jurisdictional claims in published maps and institutional affiliations.

\section{References}

1. Yokozawa T, Yokoyama A. Chain-growth polycondensation: living polymerization nature in polycondensation and approach to condensation polymer architecture. Polym J 2004;36:65-83.

2. Kamigaito M. Recent developments in metal-catalyzed living radical polymerization. Polym J 2011;43:105-120.

3. Yashima E. Synthesis and structure determination of helical polymers. Polym J 2010;42:3-16.

4. Tezuka Y. Topological polymer chemistry for designing multicyclic macromolecular architectures. Polym J 2012;44:1159-69.

5. Ouchi M, Sawamoto M. Sequence-controlled polymers via reversible-deactivation radical polymerization. Polym J 2018;50:83-94.
Makoto Ouchi

ouchi@living.polym.kyoto-u.ac.jp

1 Department of Polymer Chemistry, Graduate School of Engineering, Kyoto University, Kyoto, Japan

2 Materials Science and Engineering Department, University of Washington, Seattle, WA, USA
3 Department of Macromolecular Science, Fudan University, Shanghai, China

4 Department of Applied Chemistry and International Institute for Carbon-Neutral Energy Research (WPI-I2CNER), Kyushu University, Fukuoka, Japan 